PRAXIS

educativa

Universidad Nacional de La Pampa

Facultad de Ciencias Humanas

Instituto de Ciencias de la Educación

para la investigación interdisciplinaria

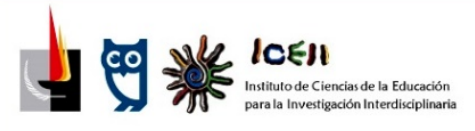

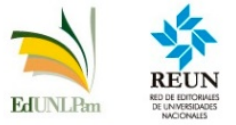

ISSN 2313-934X

SANTA ROSA, LA PAMPA, ARGENTINA

Correo electrónico: iceii@humanas.unlpam.edu.ar

Disponible en https://cerac.unlpam.edu.ar/index.php/praxis

Enseñanza virtual de la escritura de posgrado: análisis sobre la evaluación de las prácticas. Artículo de Lourdes Morán, Guadalupe Álvarez e Hilda Difabio de Anglat. Praxis educativa, Vol. 26, No 1 enero - abril 2022. E - ISSN 2313-934X. pp. 1-17.

\title{
Enseñanza virtual de la escritura de posgrado: análisis sobre la evaluación de las prácticas
}

Virtual teaching of postgraduate writing: analysis about the evaluation of practices

Ensino virtual da escrita da pós-graduação: análise sobre a avaliação das práticas

\section{Lourdes Morán}

Universidad Autónoma de Entre Ríos, Universidad de Buenos Aires, Consejo Nacional de Investigaciones

científicas y técnicas (CONICET), Argentina

moran.lourdes1@gmail.com

ORCID 0000-0003-2451-0235

\section{Guadalupe Álvarez}

Universidad Nacional de General Sarmiento, Consejo Nacional de Investigaciones Científicas y Técnicas

(CONICET), Argentina

galvarez@ungs.edu.ar

ORCID: 0000-0001-7152-730X

\section{Hilda Difabio de Anglat}

Centro de Investigaciones Cuyo, Consejo Nacional de Investigaciones Científicas y Técnicas (CONICET),

Argentina

ganglat@gmail.com

ORCID: 0000-0002-9679-1745

Recibido: 2021-06-09 | Revisado: 2021-08-09 | Aceptado: 2021-09-02 


\section{Resumen}

La enseñanza de escritura de posgrado es un eje esencial y poco presente en los estudios sobre ese nivel. Por lo general, los estudiantes evidencian grandes dificultades al momento de producir sus escritos finales. En torno a esta dificultad se han implementado distintas intervenciones, entre ellas los cursos de escritura virtual. En el presente trabajo, se comunican los resultados de un estudio cualitativo de generación conceptual focalizado en un taller virtual orientado a promover la escritura de la tesis. El análisis de este artículo está centrado en la evaluación docente acerca de las prácticas. En los resultados se sistematizan los análisis que realiza el profesorado en dos dimensiones: la primera, vinculada a la modalidad de enseñanza y los recursos tecnológicos utilizados; la segunda, relacionada con el proyecto didáctico-pedagógico. Las dimensiones construidas se tornan insumos esenciales para quienes deseen profundizar en las prácticas de la enseñanza virtuales de escritura de posgrado.

Palabras clave: escritura académica, educación a distancia, práctica pedagógica, técnica didáctica, evaluación de la educación.

\section{Abstract}

Postgraduate writing teaching is an essential subject and rarely present in studies at that level.

In general, students experience great difficulties in producing their final writings. Different interventions have been implemented around this difficulty, including virtual writing courses. In the present work, the results of a qualitative study of conceptual generation are reported. The study focuses on a virtual workshop aimed at promoting the writing of the thesis. The analysis of this article is focused on the teacher's evaluation about the practices. The results systematize the teaching analyzes in two dimensions: the first, about the teaching modality and the technological resources used; the second, about the didactic-pedagogical project. The constructed dimensions are essential inputs to deepen the virtual teaching practices of postgraduate writing.

Keywords: academic writing; distance education; teaching practice; classroom tecniques; educational evaluation.

\section{Resumo}

O ensino da escrita na pós-graduação é um eixo essencial e pouco presente nos estudos desse nível. Em geral, os alunos apresentam grandes dificuldades na produção de seus escritos finais. Diferentes intervenções têm sido implementadas em torno dessa dificuldade, incluindo cursos de redação virtual. No presente trabalho, são relatados os resultados de um estudo qualitativo de geração conceitual focado em uma oficina virtual voltada para a promoção da redação da tese. A análise deste artigo está centrada na avaliação do professor sobre as práticas. Nos resultados, as análises realizadas pelo corpo docente são sistematizadas em duas dimensões: a primeira, vinculada à modalidade de ensino e aos recursos tecnológicos utilizados; a segunda, relacionada ao projeto didático-pedagógico. As dimensões construídas tornam-se insumos essenciais para quem deseja aprofundar as práticas de ensino virtual da escrita de pósgraduação.

Palavras-chave: escrita acadêmica; educação a distância; prática pedagógica; técnica didática; avaliação educacional. 


\section{Introducción y estado de la cuestión}

La elaboración del trabajo final de las carreras de posgrado, particularmente la tesis, suele ser una de las actividades de mayor dificultad y de escasa concreción por parte del estudiantado. Frente a ello, a lo largo de los últimos años se han implementado distintas intervenciones que se orientan a dar respuesta a estas problemáticas. Desde los talleres y los seminarios de tesis bajo la responsabilidad de docentes especialistas (Arnoux et al., 2004; Carlino, 2009; Delyser, 2003) hasta los grupos de escritura (Aitchison y Lee, 2006; Colombo,2013 y Carlino, 2015; Ferguson, 2009;

Maher et al., 2008) y las retroalimentaciones (feedback) de supervisores y directores (Basturkmen et al., 2014; Li y Seale, 2007). Estos estudios abordan la intervención en el proceso de escritura buscando asistir al estudiantado en la elaboración de las tesis. De manera más reciente, se han llevado a cabo instancias de formación virtual (Alvarez y Difabio de Anglat, 2016, 2017, 2018; Difabio de Anglat y Heredia, 2013) que se orientan a asistir y acompañar en la escritura de posgrado pero en modalidad a distancia. El desarrollo de este proceso de acompañamiento a distancia ha sido escasamente investigado. Bajo la convicción de que la virtualidad no consiste sólo en un cambio de modalidad, se propuso estudiar las prácticas de enseñanza virtual de la escritura de posgrado.

Las prácticas de la enseñanza representan el conjunto de acciones que realiza el profesorado en función del proceso educativo que lleva a cabo. Como acciones concretas abarca diferentes instancias de la actuación docente. La intervención pedagógica, que ocurre en el momento del aula, representa al menos uno de los momentos. La planificación, el momento precedente y la evaluación de las prácticas, el momento posterior (Zabala, 1995).

Estudiar las prácticas educativas, desde este enfoque, exige situarse en un modelo en el que sólo se comprenden las acciones y decisiones docentes si se miran desde la complejidad. Analizar las prácticas supone establecer relaciones entre los diferentes aspectos de la realidad, comprender la intervención docente situada y considerar los diversos determinantes de la práctica en aspectos organizativos, institucionales, culturales, etc. Asimismo, supone considerar la práctica como un proceso dinámico y fluido que se ajusta en el devenir de la acción y del que sólo podemos dar cuenta al final del proceso educativo, con una mirada retrospectiva del hecho educativo. Durante la evaluación se recuperan las intenciones, las previsiones, las expectativas y se realiza la valoración de los resultados con una interpretación dinámica de lo que ha sucedido en el desarrollo de la propuesta formativa, desde su planificación hasta el final de la implementación. Desde esta perspectiva se comparte la visión de Litwin (2000) acerca de la evaluación de la práctica docente desde un enfoque didáctico. Para la autora, evaluar implica: juzgar la enseñanza y el aprendizaje, atribuir un valor a los actos y las prácticas del profesorado y a los actos que dan cuenta de los procesos de aprendizaje de los estudiantes. La valoración de los resultados, el análisis y reflexión de la situación educativa desarrollada en el aula, la valoración de los imprevistos son aspectos que impactan en la evaluación de los procesos educativos. En este sentido, la evaluación como práctica pedagógica se basa en registrar y analizar todo lo sucedido en la implementación, articularlo con los resultados alcanzados por el estudiantado y con la 
planificación realizada: desarrollar una reflexión crítica en torno a toda la actuación docente (Bain, 2006; Finkel, 2008). Consiste entonces en desplegar un proceso recursivo que vuelve el análisis al inicio del proceso, con las intenciones y metas formativas y avanza en lo sucedido, considerando las respuestas a los imprevistos y eventualidades ocurridas

El análisis de las prácticas educativas requiere de un profesional que sea capaz de mantener un diálogo reflexivo con las situaciones en las que desarrolla su actividad.

El profesorado que realiza una práctica reflexiva sobre sus propias decisiones analiza los momentos previos, de preparación de la actuación pedagógica, las instancias de desarrollo en el aula y el momento posterior de revisión de la acción. De este modo, asume un enfoque de construcción de conocimiento sobre su propia actuación en la cual teoría y práctica, previsión y realidad, intenciones y acciones se articulan indisolublemente.

Schön (1992) concibe la reflexión como un análisis que permite atribuir significado a los hechos y orienta la acción. El pensamiento práctico, sustento del profesional reflexivo, se basa en un análisis crítico de lo sucedido en la acción y durante la acción docente. Este pensamiento práctico se construye en diferentes momentos de análisis de la acción. Schön diferencia así, tres fases del pensamiento práctico: conocimiento en la acción (propia de la etapa de planificación), reflexión en y durante la acción (que sucede durante la implementación) y la reflexión sobre la acción y sobre la reflexión en la acción (característica de la etapa de evaluación). Dado que este trabajo se ocupa de la evaluación, se focaliza en la tercera fase del pensamiento práctico.

La reflexión sobre la acción y sobre la reflexión en la acción se basa en el análisis efectuado en una etapa posterior de la actuación docente. El análisis se realiza sobre los procesos y características de la acción, incluyendo en estos procesos la reflexión que se ha generado durante la práctica. En esta fase de la reflexión el docente aprende de su propia práctica, analiza con mirada crítica la multidimensionalidad de lo acontecido y da significatividad a las actuaciones desarrolladas. En su interacción con la situación, reconstruye la acción pasada desde la planificación hasta el fin de la implementación, analizando todos los elementos intervinientes. Se trata, desde el punto de vista de Schön en un conocimiento de tercer orden que analiza los dos anteriores en relación con la situación y el contexto. En esta instancia el docente pone en consideración diferentes aspectos: las características de la situación ocurrida, los procedimientos que han entrado en juego en el diagnóstico y desarrollo de la propuesta formativa, la determinación de metas, la elección de medios y la propia intervención (las decisiones tomadas), los esquemas de pensamiento, las teorías implícitas, creencias y formas de representar la realidad vivida por el profesional práctico.

Cuando el profesorado realiza la reflexión sobre prácticas educativas que involucran entornos digitales se ponen en consideración aspectos vinculados con el cambio de la modalidad.

Cuando se aborda el análisis de las prácticas educativas mediadas por las tecnologías de la información y la comunicación (TIC) y se busca reflexionar sobre la acción es esencial mirar cómo se desarrollan en la distancia toda la propuesta formativa en base a la relación entre los actores involucrados. Se concede al profesorado un papel esencial, de facilitador de experiencias e 
intercambios que permiten construir aprendizajes complejos y la generación de instancias de interacción social para la construcción con otros (Baquero, 1996; Coll, 1995; Vigotsky, 1987). La valoración de las propuestas formativas mediadas por TIC no está en los recursos tecnológicos en sí mismos, sino en los usos pedagógicos que se realice de ellos para lograr la construcción del conocimiento por parte del estudiantado y una mayor interactividad entre todos los componentes de toda la situación formativa.

Analizar la interactividad supone observar aspectos esenciales de los procesos de enseñanza y aprendizaje en la virtualidad. Algunos de esos factores son las actuaciones en torno a una tarea o contenido, las formas de organización de las actividades, los intercambios mutuos en torno a los contenidos de aprendizaje, y las ayudas que el profesorado presenta para avanzar en la construcción del conocimiento (Onrubia, 2005).

La interactividad varía según la situación educativa; del mismo modo, las ayudas docentes varían en función de las necesidades del estudiantado. Es por ello que, al reflexionar sobre las prácticas, debemos considerar varias dimensiones: lo sucedido con las actividades, las ayudas ofrecidas y la forma en que la tecnología facilitó u obturó estas acciones docentes.

Una propuesta interesante para el análisis de la interactividad es la que desarrollan Coll et al. (2008). Ambos autores construyeron tres dimensiones para analizar la interactividad de una propuesta formativa considerando dos planos: el del diseño y el de su desarrollo real (es decir, lo propuesto y lo sucedido en los hechos). Las dimensiones que consideran son las siguientes: la interactividad tecnológica potencial (la posibilidad de interactividad que ofrece la tecnología seleccionada y que se tiene en cuenta en el momento del diseño de la enseñanza), la interactividad pedagógica potencial (la posibilidad de interactividad que ofrece el planteo pedagógico y didáctico y que se tiene en cuenta en el momento diseño de la enseñanza) y la interactividad tecnopedagógica real (la vinculación efectiva entre el uso de la tecnología y el planteamiento pedagógico y didáctico propuesto). Estas dimensiones resultan ser un instrumento adecuado para evaluar la actuación docente desde una perspectiva pedagógica.

\section{Metodología}

El estudio, de carácter cualitativo de generación conceptual se centra en las prácticas de enseñanza virtual de la escritura de posgrado.

Para llevarlo a cabo, se recuperaron datos de la primera edición de un taller virtual orientado a la escritura de la tesis de posgrado. Este taller se realizó durante 2016 en la Facultad de Filosofía y Letras de la Universidad Nacional de Cuyo. Comprendió la participación de 11 estudiantes de carreras de doctorado y sus docentes, dos investigadoras. Una de ellas formada, en Ciencias de Educación con una especialización en textos académicos; la otra docente, formada en Letras y especializada en lectura y escritura académica, así como en enseñanza mediada por tecnologías. Ambas fueron las responsables del diseño de la intervención didáctica y de su implementación. 
El taller comprendió 11 semanas, en cada una de las cuales se expusieron temas y tareas específicas. Durante la primera semana, se realizaron las presentaciones y se implemnetaron las actividades de diagnóstico. En la segunda, se realizó una reflexión sobre las tareas de escritura, con foco en la redacción académica. Las siguientes siete semanas estuvieron centradas en el análisis de los paratextos y las secciones básicas de la tesis. Las tareas se orientaron a la búsqueda y al análisis de los paratextos y las distintas secciones de la tesis. Se profundizó sobre el modelo de la situación comunicativa, el modelo del evento y el modelo textual. Los ejercicios propuestos tuvieron carácter individual y en otros grupal. En todos los casos, las docentes compartieron devoluciones de las tareas realizadas. Las dos últimas semanas se dedicaron a elaborar el borrador de un capítulo (o fragmento) de la tesis, lo que representó el trabajo de evaluación final.

Aunque se había planificado utilizar la plataforma Moodle para este taller, debido a cuestiones técnicas se decidió combinar distintas herramientas de la Web: correo electrónico, blog, documentos compartidos en GoogleDrive.

En el estudio realizado se parte de la realización de este primer taller y a través de las entrevistas a las docentes se integran los aportes de las ediciones posteriores, enriqueciendo el análisis.

Para la recolección de la información se utilizaron diversos instrumentos y se procedió según se describe a continuación:

Análisis documental de la propuesta montada en el blog. Inicialmente, se realizó una lectura global del curso considerando la estructura, las partes en las que estuvo dividida, los materiales disponibles y las actividades. Se reconocieron patrones de semejanza y diferencia entre los módulos. Luego, se realizó una lectura más profunda de cada módulo y sus componentes. Se analizaron los materiales (tipo, estructura, información, intencionalidad del texto), las actividades (tipo, consigna, tarea requerida al alumnado) y los espacios de interacción docente-alumno (intervenciones, frecuencia de respuesta, contenido).

Cuestionarios de diagnóstico, de indagación de saberes y competencias, y de evaluación final sobre el curso. Estos cuestionarios fueron los que se utilizaron para el curso. Como procedimiento de análisis se realizó la misma secuencia empleada con blog del curso. Se llevó a cabo una lectura inicial, identificando patrones generales de análisis y después se realizó una lectura en profundidad. En los diferentes cuestionarios, se tuvieron en cuenta los siguientes aspectos: intencionalidad, preguntas formuladas e información recabada.

Entrevistas semiestructuradas, de preguntas orientadoras, a las docentes responsables del diseño y la implementación del curso. Las entrevistas se orientaron a explorar decisiones previas y antecedentes que hicieron posible el diseño y la implementación de la instancia formativa. Las preguntas se construyeron en función de tres ejes: a) de indagación del contexto y acerca de la experiencia previa docente, b) de concepciones docentes y c) de la planificación concreta de la propuesta. Todas las preguntas apuntaron a la reflexión del profesorado sobre las acciones y las decisiones tomadas. 
Los datos obtenidos fueron analizados de acuerdo con el método comparativo constante (Glaser y Strauss, 1967), desde la categorización del material primario hasta la generación conceptual. De esta forma, el procedimiento analítico ha comprendido:

Reconocimiento de unidades de significado y categorización inicial. En la primera fase se desarrolló el análisis documental de materiales (blog y cuestionarios) y lectura en profundidad de las entrevistas a las docentes. Para cada incidente o segmento textual de interés, se estableció una posible categoría. Luego, se determinaron categorías emergentes, se compararon los incidentes de categorías previas y se identificaron rasgos propios de cada categoría. Se cerró esta etapa con la elaboración de un documento con todas las categorías y los "incidentes" de cada una de ellas. Integración de categorías y propiedades. En esta etapa, se realizaron comparaciones entre los "incidentes" de cada categoría y entre "incidentes" de diferentes categorías conformadas. De este modo se llevó a establecer categorías más amplias y, a su vez, deconstruir algunas categorías en subcategorías.

Elaboración de la descripción genérica de la experiencia. El proceso previamente descripto culminó en un conjunto menor de categorías, pero de alto nivel, que fueron usadas para describir los elementos centrales de la experiencia.

Desarrollo de la narrativa en torno a la experiencia. En la narrativa, se integraron las descripciones en torno a las categorías construidas. Se establecieron interrelaciones entre el análisis del corpus y la categorización elaborada, dando cuenta tanto de la experiencia como de las diferentes valoraciones que sobre ella tienen las docentes. Este proceso final permitió confeccionar las conclusiones del presente artículo.

Para atenuar el grado de injerencia de las docentes en el análisis, el trabajo ha sido llevado a cabo inicialmente por una investigadora que no ha diseñado ni implementado la experiencia. Recién después de esa primera fase, las investigadoras que han participado de la experiencia han podido revisar y completar el análisis inicial. La investigadora no docente ha sido responsable de la última revisión del análisis.

\section{Análisis}

A partir del análisis e interpretación de la información recolectada se identificaron un conjunto de aspectos que se presentan durante la instancia de evaluación de las prácticas. En la mirada retrospectiva de las acciones el profesorado analiza cómo se articulan el diseño educativo planificado y la acción real ocurrida.

A partir de la mirada retrospectiva de las docentes, se identificaron dos dimensiones:

La evaluación de las prácticas en función de la modalidad de enseñanza y los recursos tecnológicos utilizados.

La evaluación de las prácticas en función del proyecto didáctico-pedagógico.

A continuación, se desarrollarán cada una de estas dimensiones. 


\section{La evaluación de las prácticas en función de la modalidad de enseñanza y recursos tecnológicos utilizados}

En esta dimensión se concentran las reflexiones en torno a las intencionalidades para la utilización de la virtualidad y lo que sucedió en la práctica. Las reflexiones docentes se cruzaron con el análisis documental de la propuesta en el blog y con las encuestas de finalización efectuadas al estudiantado

En las reflexiones docentes, la elección de la modalidad virtual y el recurso tecnológico (campus virtual) estuvo, en un primer momento, asociado a cuestiones no vinculadas con el proyecto pedagógico. Inicialmente la elección obedeció a intereses personales vinculados con la investigación de los procesos de escritura con mediación tecnológica y a las posibilidades que ofrece la virtualidad de trabajo conjunto a distancia. En una segunda instancia, la decisión se centró en las concepciones de las docentes acerca de la enseñanza y el aprendizaje en contextos virtuales.

Desde el inicio estuvo la idea de diseñar una oferta de formación virtual, lo que surgió, por un lado, por mi interés en explorar en el posgrado la enseñanza de la lectura y la escritura mediada por tecnologías digitales, tema que venía investigando en el nivel universitario de grado y pregrado. (...) Por otro lado, esta posibilidad se adecuaba a nuestras circunstancias vitales dado que vivíamos (y seguimos viviendo) en diferentes ciudades y ninguna estaba disponible como para dictar un seminario en otro lado. (Docente 1) Concebimos la enseñanza virtual como un proceso complejo en tanto las dimensiones propias de la enseñanza son "atravesadas" (actualizadas, modificadas, repensadas...) por los nuevos medios digitales. Estamos convencidas de que no se trata de subir documentos y proponer discusiones abiertas sobre ellos, sino que el diseño se debe basar en actividades de diversa naturaleza (individuales y grupales) pautadas con una frecuencia determinada. Asi, propusimos realizar un curso que comprometiera a los estudiantes con actividades frecuentes para sostener y profundizar su presencia virtual. (Docente 2)

Si bien la decisión acerca de incluir la tecnología en principio respondió a cuestiones exógenas al diseño pedagógico, luego las decisiones se concentran en la intencionalidad de formación y en los recursos más adecuados para la propuesta pedagógica que llevaba a cabo el profesorado. En las entrevistas, las docentes refieren al contexto por el cual se asumió realizar un curso virtual, pero las razones no sólo están en la distancia y en interés personal de investigación, los argumentos enfatizan las posibilidades que ofrece la virtualidad para acompañar una propuesta de enseñanza y el aprendizaje del estudiantado. Esta mirada acerca de la importancia destinada a las cuestiones pedagógicas se evidencia cuando se comparten los análisis acerca de lo sucedido en la práctica con la virtualidad: el imprevisto de tener que cambiar de recurso. 
Un aspecto fundamental a destacar es que, dado que la universidad ese año (después ya si) no contaba con la plataforma educativa habilitada para la formación de posgrado, recurrimos a una plataforma Moodle más informal. El problema fue que diseñamos gran parte del curso y dimos inicio alli al curso y, a las horas de haber comenzado, la plataforma se cayó y no pudimos recuperar lo realizado. Al instante pensamos una alternativa: utilizaríamos diferentes aplicaciones y programas particularmente de la Web, para dictar el curso. Así, buscamos las aplicaciones que presentaran las funcionalidades que pensábamos utilizar en la plataforma: las entradas de los blogs, para los intercambios grupales que habiamos pensado llevar a cabo en los foros; los documento compartidos, para el trabajo de escritura grupal que pensábamos desarrollar en las wikis; los correos electrónicos, para compartir documentos e informaciones que en la plataforma se hubieran presentado en carpetas, etiquetas o herramientas de subida de tareas. (Docente 2)

En concordancia con lo planteado, la necesidad de cambio de recurso tecnológico no resultó ser un hecho disruptivo en función de la configuración del proyecto pedagógico. Las docentes enfrentan el hecho de la "caída" de la plataforma como una eventualidad en el desarrollo de su actividad docente. Se centran pues, en buscar y seleccionar recursos tecnológicos disponibles que acompañaran la propuesta didáctico-pedagógica.

En estas reflexiones podemos observar cómo se articula la interactividad tecnológica potencial (Coll et al., 2008) con la acción docente. Si bien, la interactividad tecnológica potencial se plantea como el análisis de la posibilidad de interactividad que ofrece la tecnología seleccionada en el momento del diseño de la enseñanza, esta interactividad se reconsidera durante el desarrollo de la propuesta en función de las eventualidades surgidas. En esta línea se puede avanzar aún más en sostener que no sólo hay análisis de la interactividad tecnológica potencial durante el diseño de la enseñanza, sino también durante la práctica que se presenta dinámica y cambiante suceptible de ajustes en el devenir de la acción. (Zabala, 1995). En este sentido, plantear un cambio de la tecnología durante el transcurso de la formación supone reconsiderar las posibilidades y restricciones que ofrecen los recursos tecnológicos para la propuesta pedagógica, volver a analizar la interactividad tecnológica potencial de los nuevos recursos. Involucra, además, de esta manera la reflexión sobre la acción. La reflexión que el profesorado realiza en el transcurso de su acción y que le permite adecuar sus prácticas a las nuevas circunstancias (Schon, 1992).

A partir del análisis de las expresiones del estudiantado se observó que no hay percepción acerca del cambio de tecnología. En las expresiones recolectadas no se encuentran menciones que se dirijan al cambio de recurso o sobre las decisiones que realizan las docentes en función de la interactividad tecnológica potencial. Sus valoraciones se dirigen más hacia la propuesta pedagógica potenciada por la tecnología, que al cambio de recurso y las decisiones docentes involucradas en esta dimensión. En este punto la apreciación que realiza el estudiantado no se 
fundamenta en la introducción de recursos por sí mismos, sino en el aporte que ellos brindan a la propuesta pedagógica. Este eje de análisis coincide con la perspectiva del profesorado.

Efectivamente las características de las TIC por sí solas no garantizan el aprendizaje, ya que éste depende, esencialmente de la calidad de la propuesta educativa. El estudiantado no percibió los cambios en los recursos porque la elección obedeció a criterios pedagógicos. La cuestión tecnológica no ha sido un elemento distractor en el proceso de enseñanza y de aprendizaje.

\section{La evaluación de las prácticas en función del proyecto didáctico-pedagógico}

El segundo eje de análisis realizado en relación con la evaluación se concentra en aspectos vinculados con el proyecto didáctico-pedagógico. En el análisis de la información recolectada de las entrevistas docentes y los cuestionarios estudiantiles, los aspectos que se presentan con mayor recurrencia están vinculados con:

El cumplimiento de los objetivos propuestos y su superación en términos de expectativas. Las actividades desarrolladas centradas en la organización grupal y de modalidad práctica. Las intervenciones docentes que obedecen a un claro rol de facilitador del aprendizaje

El cumplimiento de objetivos es uno de los aspectos más significativos que los actores implicados mencionan en sus evaluaciones. En este aspecto se involucran análisis sobre las expectativas en relación al curso, los aprendizajes logrados y las oportunidades de práctica otorgadas al estudiantado.

La evaluación del profesorado reúne las intencionalidades de formación delineadas durante el diseño y el grado de alcance durante el desarrollo hasta la finalización del curso. Como elemento de análisis de esta evaluación se considera el grado de avance del estudiantado desde el punto de partida. No se centran en los mismos parámetros para todos, por el contrario se considera el nivel alcanzado por cada uno de ellos.

Algunos de los siguientes fragmentos ilustran esta posición:

Por el referido destinatario de este curso específico, pensamos que necesitaban una propuesta curricular que "siguiera" la estructura de una tesis que se ajustara a cada perfil y a lo que conocían acerca de la escritura -desde el indice hasta las referencias bibliográficas- y que trabajara con textos auténticos, con "tesis". En este sentido, buscamos generar una propuesta que combinara actividades individuales con actividades colaborativas, que promovieran la reflexión, la metacognición sobre el propio proceso, la argumentación, la justificación en su disciplina y en la investigación de nivel de posgrado, todo ello desde el punto de partida de cada uno de los participantes. Todo ello encaminado a un producto tangible: la escritura de un capítulo (o de un apartado) de la tesis -para buena parte de los estudiantes ha sido el primero. (Docente 2) Como principal logro, percibido sobre todo a través de la voz de los estudiantes, podemos destacar que algunos inician la escritura de la tesis o realizan avances importantes en este sentido. Cada uno según su recorrido, respetando su etapa en el proceso de investigación y de escritura (Docente 1) 
En las expresiones del estudiantado se destaca el logro en términos de aprendizajes relacionados con la comprensión y la producción desarrollada.

Me pareció súper claro y que cubrió perfectamente los objetivos planteados al inicio. El material de lectura es sencillo, concreto y súper claro. El paso a paso me resultó excelente! Había hecho otros de escritura académica pero no eran tan concretos y con pautas bien dirigidas a la elaboración de la tesis. (Estudiante 4)

Sin adular, creo que es uno de los más útiles entre los cursos generales de las carreras de doctorado en la facultad. (...) el taller nos proporcionó bibliografía de referencia sobre producción del discurso científico (en particular, de producción de tesis) y estimuló la reflexión. Dejó muy clara la idea-eje de que la escritura no es algo mecánico y formal, sino que es un modo de configurar el trabajo intelectual. (Estudiante 8)

En esta dimensión de análisis, la evaluación como práctica pedagógica se centra en analizar lo sucedido en la implementación articulada con los resultados alcanzados por el alumnado y con la planificación realizada. La mirada acerca de la evaluación en términos de objetivos se realiza en forma global, no analiza el aporte de cada una de las actividades por el contrario hace un análisis de toda la propuesta en su conjunto. Vincula la intencionalidad con los resultados en términos de logros alcanzados en forma particular por cada participante. Desde el análisis de Ken Bain (2006) y Don Finkel (2008) representaría una reflexión crítica en torno a la actuación docente en su conjunto.

El segundo aspecto destaca las actividades desarrolladas en el curso. Las menciones de los diferentes actores se agrupan en dos dimensiones centrales acerca de la evaluación de las actividades. Por una parte, se enfatiza el agrupamiento estudiantil durante las propuestas. Por otra parte, se prioriza la actividad de producción y de reflexión.

En cuanto al tipo de agrupamiento, el análisis realizado destaca una clara priorización de las actividades grupales por sobre las individuales. La grupalidad aparece como una posibilidad de potenciar el trabajo, enriquecer la producción individual y sentirse acompañado en el proceso de aprendizaje. Cada una de las propuestas diseñadas contiene instancias de intercambio o actividad grupal. En las consignas y en los foros de intercambio se refleja esta condición. Una de las consignas de los foros plantea: "Es importante que compartan dudas y preguntas porque esto será fundamental para el buen desarrollo de los contenidos. La discusión entre los participantes enriquece en su esencia al aprendizaje."

En las expresiones docentes se evidencia de la siguiente manera:

Leer las producciones de otros compañeros, intercambiar ideas y reflexiones son actividades grupales de gran valor (...). También creemos que el trabajo en grupo de pares y expertos representa un aporte de esta formación en tanto los estudiantes perciben asi que no se encuentran solos en la tarea de investigación y escritura, que hay otros en 
situaciones similares, con los cuales pueden interactuar y aprender. Con el taller se abre un espacio de diálogo académico que excede el tiempo del curso. (Docente 2)

En las expresiones del estudiantado la grupalidad también se presenta como un elemento destacable en la propuesta.

El trabajo en grupo fue muy productivo en la medida que pudimos intercambiar ideas y consensuar a la hora de realizar las actividades. Ha sido una instancia motivadora y que me ha proporcionado más seguridad (Estudiante 7)

El trabajo en grupo me ayudó a dar un paso atrás frente a un análisis y mirarlo de nuevo, reverlo. Creo que esto es muy importante en el análisis de textos, contar con una segunda o más perspectivas (la del informante, la de un colega) (Estudiante 1)

Las prácticas aparecen "atravesadas" por una didáctica en grupos (Souto, 2000). En las actividades el aspecto relacional adquiere un gran valor. En esas instancias se enriquece el trabajo y se potencia el aprendizaje. Se abre la posibilidad de intercambiar perspectivas, poner a disponibilidad de los otros lo que cada uno sabe, conoce y comprende. Desde una perspectiva psicológica, la dinámica en grupo posibilita también reducir el sentimiento de encontrarse solo ante el aprendizaje de un saber nuevo. La grupalidad permite reducir la ansiedad de sentirse solos en los procesos de aprendizaje (Romero y Saune, 1995). La dinámica de grupos, en este curso adquiere un doble carácter. Por una parte permite enriquecer el aprendizaje estudiantil al compartir lo que se conoce o desconoce acerca de un saber; por otra parte, permite reducir la ansiedad de encontrarse solo ante el proceso de escribir. Sin embargo, es importante señalar que la conformación de grupos no está exenta de dificultades. En términos de consolidación grupal, en ocasiones, es difícil para los estudiantes gestionar el tiempo necesario para el trabajo grupal y lograr el nivel de compromiso que requiere la tarea con otros participantes a los que no conocen con profundidad.

El segundo aspecto distintivo de las actividades refiere a la priorización de la producción. La secuencia de actividades propuestas enfatiza la acción de realización práctica por parte del estudiantado junto a la reflexión acerca de la propia escritura. Se incentiva la mirada crítica durante el proceso de escritura y la capacidad de reflexión y análisis sobre la producción realizada. En el análisis de las actividades, gran parte de ellas contienen consignas orientadas a promover la reflexión metacognitiva respecto del proceso personal de producción.

En las expresiones del profesorado la secuencia se describe de la siguiente manera: Los estudiantes siguen la propuesta didáctica, la que tiene una organización bastante pautada: 10) leen la bibliografía sugerida: el libro de cabecera; $2^{\circ}$ ) van completando las guías de actividades en los tiempos previstos y según la modalidad propuesta (individual, grupa, en el blog, o en Word) a propósito de los capítulos de tesis proporcionados en los dos primeros módulos, desde el tercero (capítulo teórico) trabajan sobre tesis de su 
especialidad (una que cada alumno o grupo seleccione especialmente; inclusive, en algunos casos, la propia tesis); 30) van reflexionando "harto" (como se dice en Chile), es la actividad concomitante de todo el taller; obviamente, algunos se comprometen más con las actividades y se dedican más a dicha reflexión que otros.(Docente 1)

En relación con la secuencia, el estudiantado señaló:

No recuerdo una actividad de este curso en la cual no haya tenido que sentarme a pensar un buen rato antes de intentar darle respuesta. Fue un desafío muy interesante. Las actividades me permitieron la reflexión y de la toma de conciencia acerca de las propiedades retóricas y lingüísticas de estas secciones para transferir la comprensión lograda a la producción de mi tesis. (Estudiante 8).

La lectura de buenos textos me ayudó a pensar y a valorar más críticamente lo que escribí. Hice análisis de texto como de "disecar" un bicho. Definitivamente me sacó muchos de los miedos con los que empecé el curso. Creo que es un proceso de maduración... jLo empezaría de nuevo! (Estudiante 5)

La secuencia de actividades propuestas recupera las características del pensamiento práctico de Schön (1992). La reflexión como un proceso de análisis permite atribuir significado a los hechos y orienta la acción. De este modo, al reflexionar sobre la propia práctica se desarrolla un análisis crítico de lo sucedido: las decisiones asumidas, los fundamentos de la acción, los momentos de incertidumbre, etc. El pensamiento práctico se construye durante el proceso de escritura y al volver con acciones de revisión, superación y mejora de la propia producción. Las docentes enfatizan este procedimiento y el estudiantado valora en sus expresiones la posibilidad de realizar esta misma actividad de producción y reflexión.

Finalmente, se identifica al intercambio como parte esencial del desarrollo de las estrategias docentes. Se concibe en la práctica la importancia de mantener una comunicación fluida con el estudiantado y la intervención docente en función de orientar y monitorear el aprendizaje.

En las expresiones del profesorado la interacción se describe de la siguiente manera:

Todas nuestras participaciones tuvieron como eje central orientar la práctica y profundizar el análisis. Consideramos importante acompañar a los estudiantes mediante nuestras intervenciones y los intercambios constantes con ellos". (Docente 1). "Nuestras intervenciones se orientaron a valorar el desempeño de los estudiantes y alentarlos; sistematizar lo realizado, explicitando el vínculo entre los comentarios de los estudiantes; sistematizar el análisis; señalar problemas en el análisis; orientar el análisis; extender el análisis; promover la participación y derivar recomendaciones. (Docente 2)

Por su parte, el alumnado destacó: 
El modo de corrección de las profesoras me ayudó mucho. A través de gestos y sutilezas léxicas supieron brindarme la confianza necesaria para no tener temor a consultar y a equivocarme. Estuvieron siempre presentes (...) percibi un apoyo constante y firme, de un guía afectuoso dispuesto a asistirme en todo momento. (Estudiante 2)

Durante el curso recibimos devoluciones puntuales, sumamente detalladas y alentadoras. Las devoluciones eran muy buenas: a tiempo, bien dirigidas, exhaustivas, con explicaciones claras, focalizadas en el tema, con la información necesaria para esclarecerlo y motivadoras para seguir pensando. (Estudiante 4)

En este punto del análisis las reflexiones de los actores valoran las ayudas que se ofrecen para la construcción del conocimiento. La interactividad pedagógica potencial (Onrubia, 2005) que consiste en la posibilidad de interactividad que ofrece el planteo pedagógico y didáctico se presenta en el marco de la implementación real. La interactividad pensada y diseñada en el momento de la planificación se traduce en actividades y en intervenciones concretas realizadas durante el momento de la implementación. En las actividades se adecuan las intervenciones en función de la secuencia didáctica, tiempos y tipos de tareas. En esta instancia las relaciones mutuas entre estudiantes, contenido y docentes configuran el tipo de interactividad que se lleva a cabo. En el caso del curso, y en función de las expresiones de los actores, la interactividad asume forma de "andamiaje" (Bruner, 1988) de los procesos de construcción del conocimiento. El profesorado se desempeña como facilitador del proceso, retoman los aportes acerca del saber y ofrece las ayudas necesarias para completar, corregir y/o asistir en la construcción del conocimiento. En la actividad grupal el alumnado también actúa bajo la estructura de "andamiaje". A partir de las reflexiones conjuntas, los intercambios acerca de los contenidos y las acciones de producción compartida, se brindan los apoyos temporarios de los conocimientos, hasta que los saberes ya han sido adquiridos.

En el cierre de este análisis queda mencionar que los aspectos señalados corresponden a los elementos que se presentan con mayor frecuencia en la mirada retrospectiva de las docentes acerca del curso realizado. Estos elementos agrupados en las dos dimensiones construidas de la evaluación de las prácticas: en función de la modalidad de enseñanza y recursos tecnológicos utilizados y en función del proyecto didáctico-pedagógico, son sólo una parte de la evaluación de las prácticas. Desde una mirada compleja, pueden abordarse otras dimensiones.

\section{Conclusiones}

Estudiar las prácticas de la enseñanza como acciones concretas de la actuación docente es un campo de estudio interesante. En el caso abordado, la intervención pedagógica fue analizada desde la mirada retrospectiva, en la instancia de la evaluación de las prácticas de la enseñanza, en el momento posterior a la implementación. (Zabala, 1995)

Dos conclusiones relevantes interesan aportar al final de este trabajo. Por una parte, la relevancia que adquiere la reflexión como un análisis que permite atribuir significado a los hechos 
y orienta la acción Schön (1992). En el caso abordado las reflexiones sobre la acción desarrollada toman una relevancia mayor en relación con las reflexiones sobre la reflexión en la acción. En los relatos el profesorado recupera en mayor medida la mirada retrospectiva de lo sucedido durante la acción, y con menor extensión las reflexiones que surgieron durante el proceso de implementación. Las reflexiones sobre la acción priman ante las reflexiones acerca de la reflexión en la acción. Seguramente este énfasis se vincula con el tiempo transcurrido entre el curso realizado y el análisis de lo sucedido. Como sostiene Schön (1992) cuanto más tiempo transcurre entre los análisis docentes acerca de las reflexiones surgidas en la acción y el análisis de ella, mayores son las posibilidades de no observar el hecho con claridad.

Por otra parte, es interesante recuperar que en todos los relatos se le otorga mayor relevancia al proyecto didáctico-pedagógico que al tecnológico. Si bien la tecnología es un medio que se percibe como relevante, las decisiones acerca de las herramientas se asumen en función de las decisiones pedagógicas. En la experiencia recorrida la interactividad tecnológica potencial y la interactividad pedagógica potencial (Coll et al., 2008) se articulaban, inicialmente, de un modo que en la implementación requirió de ajustes. La interactividad tecnopedagógica real fue resuelta al apelar a diferentes herramientas tecnológicas, no previstas inicialmente, que permitieron una vinculación real entre el uso de la tecnología y el planteamiento pedagógico y didáctico propuesto. La configuración de prácticas de enseñanza con tics implica una mirada en la que la tecnología es intrínseca al proyecto pedagógico.

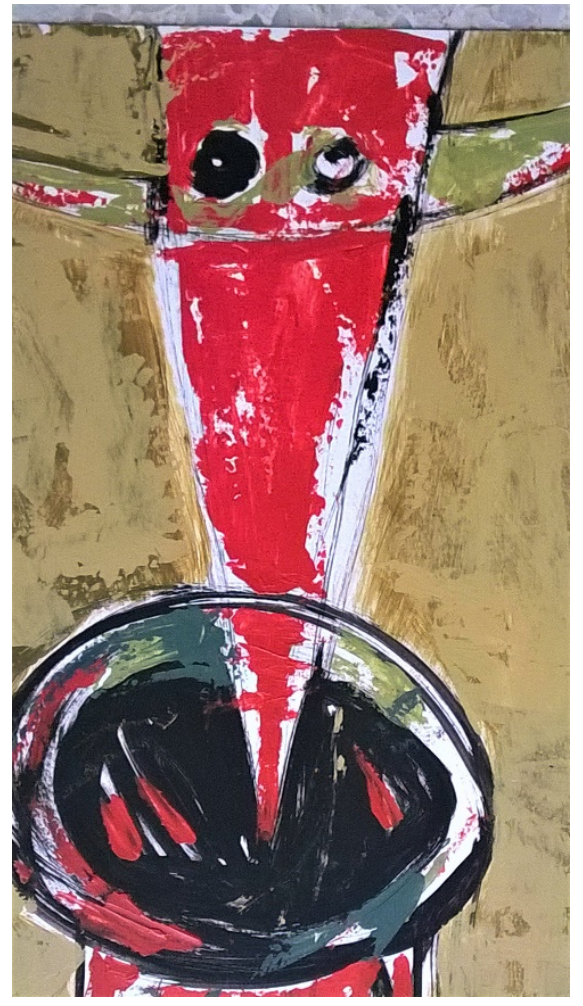

ST, acrílico. Noemí Fiscella 


\section{Bibliografía}

Aitchison, C., \& Lee, A. (2006). Research writing: Problems and pedagogies. Teaching in Higher Education, 11(3), 265-278. DOI: 10.1080/13562510600680574

Álvarez, G., \& Difabio de Anglat, H. (2018). Retroalimentación docente y aprendizaje en talleres virtuales de escritura de tesis. Revista Apertura, 10(1), 8-23. Disponible en

http://www.udgvirtual.udg.mx/apertura/index.php/apertura/article/view/996/900

Álvarez, G., \& Difabio de Anglat, H. (2017). La actividad metalingüística en espacios de interacción entre pares: reflexiones en torno a un taller virtual orientado a la escritura de la tesis de posgrado. Perfiles educativos, XXXIX (155), 51-67. Disponible en http://www.iisue.unam.mx/perfiles/perfiles_articulo.php?clave=2017-155$51-67$

Álvarez, G., \& Difabio de Anglat, H. (2016). Formación virtual en estrategias para la producción conceptual y escrita en el posgrado en Ciencias Sociales y Humanas. Revista $Q, 10$ (20). Disponible en http://revistaq.upb.edu.co/articulos/ver/543

Arnoux, E., Borsinger, A., Carlino, P., di Stefano, M., Pereira C., \& Silvestri, A. (2004). La intervención pedagógica en el proceso de escritura de tesis de posgrado. Revista de la Maestría en Salud Pública, 2(3), 1-16. Disponible en https://www.aacademica.org/paula.carlino/169

Bain, K. (2006). Lo que hacen los mejores profesores de universidad. Universitat de València.

Baquero, R. (1996). Vigotsky y el aprendizaje escolar (Vol. 4). Aique.

Basturkmen, H., East, M., \& Bitchener, J. (2014). Supervisors' on-script feedback comments on drafts of dissertations: socialising students into the academic discourse community. Teaching in Higher Education, 19 (4), 432-445. DOI: 10.1080/13562517.2012.752728

Bruner, J. (1988). Realidad mental y mundos posibles (Vol. 19962001). Gedisa.

Carlino, P. (2009). Exploración de géneros, diarios de tesis y revisión entre pares. EN Arnoux, E. (Dir.), Escritura y producción de conocimientos en las carreras de posgrado (pp. 227-246). Santiago Arcos Editor. http://www.scielo.org.mx/pdf/redie/v10n1/v10n1a1.pdf Coll, C. (1995). Elementos para el análisis de la práctica educativa. Psicología de la Instrucción III. Nuevas perspectivas. Síntesis.

Coll, C., Maurí, T., \& Onrubia, J. (2008) El análisis de los procesos de enseñanza y aprendizaje mediados por las TIC: una perspectiva constructivista. En Barberá, E., Maurí, T., \& Onrubia, J. (Coords.) Cómo valorar la calidad de la enseñanza basada en las TIC: pautas e instrumentos de análisis. Graó

Colombo, L. (2013). Una experiencia pedagógica con grupos de escritura en el posgrado. Aula Universitaria, 15, 61-68. Disponible en https://ri.conicet.gov.ar/handle/11336/12283

Colombo, L., \& Carlino, P. (2015). Grupos para el desarrollo de la escritura científico-académica: Una revisión de trabajos anglosajones. Lenguaje, 43(1), 13-34. Disponible en http://www.scielo.org.co/pdf/leng/v43n1/v43n1a02.pdf

Delyser, D. (2003). Teaching graduate students to write: a seminar for thesis and dissertation writers. Journal of Geography in Higher Education, 27(2), 169-181. D0I: 10.1080/03098260305676

Difabio de Anglat, H., \& Heredia, M. del V. (2013). El taller de tesis doctoral en educación desde un enfoque comprehensivo de escritura a través de la plataforma Moodle. En $6^{\circ}$ Seminario Internacional de Educación a Distancia. Disponible en

http://www.uncu.edu.ar/seminario_rueda/upload/t234.pdf 
Ferguson, T. (2009). The 'Write' Skills and More: A Thesis Writing Group for Doctoral Students. Journal of Geography in Higher Education, 33(2), 285-297. doi: 10.1080/03098260902734968

Finkel, D. (2008). Dar clase con la boca cerrada. Universitat de València.

Glaser, B. G., \& Strauss, A. L. (1967). The discovery of grounded theory: strategies for qualitative research.

Aldine de Gruyter.

Litwin, E. (2000). La educación a distancia. Amorrortu.

Maher, D., Seaton, L., McMullen, C., Fitzgerald, T., Otsuji, E., \& Lee, A. (2008). 'Becoming and being writers': the experiences of doctoral students in writing groups. Studies in Continuing Education, 30(3), 263-275. DOI:

$10.1080 / 01580370802439870$

Onrubia, J. (2005). Aprender y enseñar en entornos virtuales: actividad conjunta, ayuda pedagógica y

construcción del conocimiento. Revista de Educación a Distancia (RED). Recuperado a partir de

https://revistas.um.es/red/article/view/24721

Romero, R. R., \& Saune, S. T. (1995). Grupo: objeto y teoría. Lugar

Schön, D. A. (1992). La formación de profesionales reflexivos: hacia un nuevo diseño de la enseñanza y el

aprendizaje en las profesiones. Ministerio de Educación y Ciencia.

Schön, Z., \& Guerra, S. (1998). El profesional reflexivo. Publicación académica de la Universidad de la Marina

Mercante, 27.

Souto, M. (2000). Las formaciones grupales en la escuela. Paidós.

Vigotsky, L. S. (1987). Historia del desarrollo de las funciones psíquicas superiores. Editorial Científico-Técnica. Zabala Vidiella, A (1995). La práctica educativa: cómo enseñar. Gráo 